\title{
The relationship between diet quality and the severity of household food insecurity in Canada
}

\author{
Joy Hutchinson ${ }^{1}$ and Valerie Tarasuk ${ }^{2, *}$ (1) \\ 'School of Public Health Sciences, University of Waterloo, Waterloo, Canada: ${ }^{2}$ Department of Nutritional Sciences, \\ University of Toronto, Temerty Faculty of Medicine, 1 King's College Circle, Toronto, ON M5S 1A8, Canada
}

Submitted 10 February 2021: Final revision received 11 August 2021: Accepted 20 September 2021: First published online 23 September 2021

\begin{abstract}
Objective: To examine the relationship between the dietary quality of Canadian children and adults and household food insecurity status.

Design: Dietary intake was assessed with one 24-h recall. Households were classified as food secure or marginally, moderately or severely food insecure based on their responses to the Household Food Security Survey Module. We applied multivariable analyses of variance to determine whether \% energy from ultraprocessed foods, fruit and vegetable intake, Healthy Eating Index (HEI) scores, macronutrient composition and micronutrient intakes per $1000 \mathrm{kcal}$ differed by food insecurity status after accounting for income, education and region. Analyses were run separately for children 1-8 years and 9-18 years and men and women 19-64 years of age.

Setting: Ten provinces in Canada.

Participants: Respondents to the 2015 Canadian Community Health SurveyNutrition, aged 1-64 years, with complete food insecurity data and non-zero energy intakes, $N 15909$.

Results: Among adults and children, \% energy from ultra-processed foods was strongly related to severity of food insecurity, but no significant trend was observed for fruit and vegetable intake or HEI score. Carbohydrate, total sugar, fat and saturated fat intake/1000 kcal did not differ by food insecurity status, but there was a significant negative trend in protein/1000 kcal among older children, a positive trend in $\mathrm{Na} / 1000 \mathrm{kcal}$ among younger children and inverse associations between food insecurity severity and several micronutrients/1000 kcal among adults and older children.

Conclusions: With more severe household food insecurity, ultra-processed food consumption was higher, and diet quality was generally lower among both adults and children.
\end{abstract}

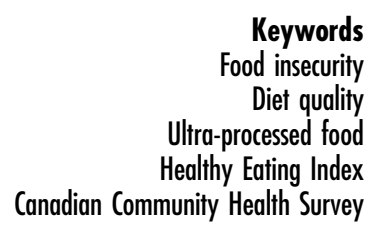

Household food insecurity is a pervasive problem in many affluent countries, including Canada. In 2017-2018, $12.7 \%$ of Canadian households reported some food insecurity $^{(1)}$, and the rate appears to have risen through the COVID-19 pandemic $^{(2)}$. Household food insecurity is a well-established social determinant of health in Canada, associated with a myriad of adverse health outcomes among children ${ }^{(3,4)}$ and adults ${ }^{(5-12)}$. The relationship between household food insecurity and measures of ill health is graded, with more severe food insecurity associated with greater likelihood of physical and mental health problems ${ }^{(12-14)}$, hospitalisation $^{(15-17)}$ and premature death $^{(18,19)}$. The pathways through which household food

insecurity impacts health and chronic disease management are not well understood, but among them is likely the effect of food insecurity on dietary intakes.

Several studies in high-income countries have reported the greater likelihood of sub-optimal dietary intakes among adults and children in food-insecure households ${ }^{(20-29)}$. Analyses of a population dietary intake survey conducted in Canada in 2004 revealed heightened nutritional vulnerability among older children, adolescents and adults in food-insecure households ${ }^{(28,30,31)}$. A subsequent comparison with US data suggested that food insecurity was a stronger marker of nutrient inadequacies in Canada than in the $\mathrm{USA}^{(29)}$. A second population survey conducted in 
2015 revealed both positive ${ }^{(32-36)}$ and negative ${ }^{(34,35,37,38)}$ changes in the nutritional quality of Canadians' dietary intakes. To date, there has been no comprehensive analysis of dietary intakes in relation to household food insecurity status using the more recent data, but food insecurity has been negatively associated with several indicators of diet quality in studies of specific population subgroups (e.g. school-aged children) ${ }^{(35,39-41)}$. These studies have all treated food insecurity as a simple binary variable, but US research has revealed greater nutritional vulnerability among individuals exposed to more severe household food insecurity (a condition labelled 'very low food security' in the USA) $)^{(21,27,42)}$.

As household food insecurity rises in Canada ${ }^{(2)}$ and the Canadian government launches small-scale market and voucher programmes to improve vulnerable households' access to fresh produce ${ }^{(43)}$, current nationally representative data on the nutritional implications of food insecurity are needed. The much more negative associations between severe food insecurity and health in $\operatorname{Canada}^{(12,15,16,18,19,44)}$, coupled with evidence of the extreme financial hardships that define this condition ${ }^{(45-48)}$, suggest that food intakes may be particularly compromised in severely foodinsecure households. Drawing on 24-h dietary intake recall data from the population survey conducted in 2015, the objective of the current study was to examine the relationship between the dietary intakes of Canadian children and adults and their household food insecurity status, considering multiple indicators of diet quality and applying a four-level categorical variable ${ }^{(49)}$ to differentiate the severity of household food insecurity.

\section{Methods}

\section{Study sample}

The current study used data from the 2015 nutritionfocused Canadian Community Health Survey (CCHS 2015 Nutrition) (n 20 487), a nationally representative survey of Canadians 1 year of age and older, living in the ten provinces ${ }^{(50)}$. This survey consisted of an in-person interview during which participants completed a 24-h dietary intake recall and general health questionnaire and had their height and weight measured. About one-third of participants completed a second 24-h recall 3-10 d later. Full-time members of the Canadian Armed Forces, people living in the territories, on First Nations communities, in institutions and in certain remote areas, and people experiencing homelessness were excluded from the survey ${ }^{(50)}$.

The current study focused on individuals aged 1-64 years who completed the first 24-h recall, excluding individuals with missing food insecurity data and those with zero energy intakes. Additionally, we excluded adults 65 years and older because of the very low prevalence of food insecurity among this age group, a phenomenon that has been linked to the protective effects of the public old-age pension programme in Canada ${ }^{(51)}$. We also excluded women who reported being pregnant or breastfeeding because we were unable to apply the Healthy Eating Index to this group. We excluded children who consumed breastmilk because of our inability to quantify intakes from this source. Our final sample size was 15909.

\section{Housebold food insecurity status}

Household food insecurity status was assessed with the eighteen-item Household Food Security Survey Module (HFSSM $^{(52)}$, completed by the most knowledgeable member of the household 18 years and older. This module, developed by the US Department of Agriculture to monitor food insecurity in that country, captures a gradient of experiences ranging from worry about running out of food, to compromising the quality of food consumed, to reducing the amount of food consumed or skipping meals, with all questions specifying that the reason for compromise is due to a lack of money ${ }^{(53)}$. The HFSSM refers to the previous 12 months, with ten questions about adults' experiences and eight questions referring to children's experiences. We categorised household food insecurity status as a fourlevel variable based on the number of affirmative responses, applying Canada's coding thresholds to define marginal, moderate and severe food insecurity as outlined in Table 1.

\section{Dietary recall data}

The 24-h dietary intake recalls were conducted by trained interviewers using an adaptation of the US Department of

Table 1 Determination of household food security status, based on the household food security survey module*

\begin{tabular}{|c|c|c|c|}
\hline Status & Interpretation & $\begin{array}{l}\text { Ten item adult food } \\
\text { security scale }\end{array}$ & $\begin{array}{l}\text { Eight item child food } \\
\text { security scale }\end{array}$ \\
\hline $\begin{array}{l}\text { Food secure } \\
\text { Marginal food } \\
\text { insecurity }\end{array}$ & $\begin{array}{l}\text { No report of income-related problems of food access. } \\
\text { Some indication of worry or an income-related } \\
\text { barrier to adequate, secure food access. }\end{array}$ & $\begin{array}{l}\text { No items affirmed } \\
\text { Affirmed no more than } \\
1 \text { item on either scale }\end{array}$ & No items affirmed \\
\hline $\begin{array}{l}\text { Moderate food } \\
\text { insecurity }\end{array}$ & $\begin{array}{l}\text { Compromise in quality and/or quantity of food } \\
\text { consumed by adults and/or children due to a lack of } \\
\text { money for food. }\end{array}$ & 2 to 5 positive responses & 2 to 4 positive responses \\
\hline $\begin{array}{l}\text { Severe food } \\
\text { insecurity }\end{array}$ & $\begin{array}{l}\text { Disrupted eating patterns and reduced food intake } \\
\text { among adults and/or children. }\end{array}$ & $\begin{array}{l}6 \text { or more positive } \\
\text { responses }\end{array}$ & $\begin{array}{l}5 \text { or more positive } \\
\text { responses }\end{array}$ \\
\hline
\end{tabular}

*Based on Canada's classification system for this module ${ }^{(49)}$. 
Agriculture's Automated Multiple Pass Method ${ }^{(50)}$. Among children 1-5 years of age, parents or guardians completed the interview by proxy. Children aged 6-11 years completed the interview with their parent or guardian, and individuals aged 12 years and older completed the interview individually ${ }^{(50)}$. For the current study, we used the first 24-h recall completed by/for each participant. Food and nutrient composition data were obtained from the 2015 Canadian Nutrient File ${ }^{(50)}$.

\section{Indicators of dietary quality}

The healthfulness of food selection was assessed by three measures closely related to Canada's Dietary Guidelines $^{(54)}$ : (1) The Canadian Healthy Eating Index $(\mathrm{HEI}-\mathrm{C})^{(55)}$ provided a composite index of diet quality. The HEI-C is based on the 2007 Canada's Food Guide; it has eleven subgroup scores which include adequacy and moderation components and sum to a maximum possible score of $100^{(55)}$. Adequacy is based on the number of servings of food groups consumed; moderation is assessed by comparing saturated fat, $\mathrm{Na}$, solid fats, alcohol and sugar intakes to prescribed thresholds ${ }^{(55)}$; (2) fruit and vegetable intake was assessed by applying the definitions of servings provided in the 2007 Canada's Food Guide ${ }^{(56)}$ and (3) the percent of energy from ultra-processed foods was determined by applying the Nova classification ${ }^{(57,58)}$ to all foods and beverages in the Food and Ingredient Details file and associated Nutrition Survey System food codes assigned to foods and ingredients in CCHS 2015 Nutrition $^{(58)}$. Although this classification system differentiates unprocessed or minimally processed foods, processed culinary ingredients, processed foods and ultra-processed food and drink products, the latter category has been most consistently associated with adverse health outcomes ${ }^{(59-61)}$. We therefore collapsed the first three categories to create a binary variable and expressed ultra-processed food intake as a percent contribution to total energy.

We also assessed individuals' intakes of macronutrients, saturated fat, total sugar, dietary fibre, $\mathrm{Na}$ and a broad spectrum of micronutrients, including vitamin A, vitamin $\mathrm{C}$, vitamin $\mathrm{D}$, thiamin, riboflavin, niacin, vitamin $\mathrm{B}_{6}$, folate, vitamin $\mathrm{B}_{12}$, Ca, Fe, Mg, P, K and Zn because they were previously associated with food insecurity in Canada ${ }^{(28)}$. Because we were interested in the composition of the diet rather than absolute nutrient intakes, we standardised micronutrients and dietary fibre per $1000 \mathrm{kcal}$ and expressed protein, fat, saturated fat, carbohydrates and total sugar as percent of total energy.

\section{Socio-demographic characteristics}

Covariates for education, income and region of residence were included in multivariable analyses because these demographic characteristics are related to household food insecurity status ${ }^{(46)}$ and self-reported dietary intakes ${ }^{(62-66)}$ in Canada. We applied a three-level categorical variable denoting the highest level of educational attainment in the household (defined as high school or less, some postsecondary certification or university degree), assigning respondents with missing data to the modal category. We also included a binary variable to differentiate households in the lowest income quintile from others. Household income quintiles were determined for the sample using self-reported income adjusted for household size by dividing by the square root of the number of people in the household to account for economies of scale. Statistics Canada provided imputed values for all households with missing income information. To capture major geographic differences in the sample, region was defined as the Atlantic provinces (Newfoundland, Nova Scotia, New Brunswick and Prince Edward Island), Quebec, Ontario, the Prairies (Manitoba, Saskatchewan and Alberta) or British Columbia.

\section{Statistical analyses}

All analyses were stratified by age and sex (among adults), grouping children 1-8 years of age, children 9-18 years of age, males 19-64 years of age and females 19-64 years of age to facilitate the exploration of potential age and sex differences. Prior Canadian research has revealed differences in the extent and nature of nutritional vulnerability associated with moderate/severe household food insecurity across age-sex groups ${ }^{(28)}$. Additionally, several studies have documented greater compromises in the self-reported diets of adults than children in food-insecure households ${ }^{(67,68)}$. Our interest in examining diet quality within age-sex groups had to be balanced by the need to retain sufficient observations within groups to compare intakes by severity of household food insecurity. We separated children by age rather than by sex because prior Canadian research suggested that the relationship between household food insecurity and children's dietary intakes differed more by age than $\operatorname{sex}^{(28)}$.

Our analysis focused on a comparison of group mean intakes from one 24-h recall by food insecurity status within these age and sex groupings. The mean of the observed intakes on a single 24-h recall can be considered an unbiased estimate of the mean usual intake of the group ${ }^{(69)}$, assuming that the survey design has considered seasonality and weekday, as was done in CCHS 2015 Nutrition.

Socio-demographic characteristics and dietary intake variables were summarised by household food insecurity status as proportions for categorical variables and means with standard errors for continuous variables. Socio-demographic characteristics were compared by household food insecurity status within age/sex groups using $\chi^{2}$ tests. We compared all dietary intake variables by household food insecurity status using ANOVA, running separate models for each age-sex stratum. Our initial models adjusted for age and sex among the two groups of children and age among the two adult groups. These 
models were then further adjusted for household sociodemographic characteristics to determine how diet quality related to severity of household food insecurity independent of relevant confounders. To satisfy ANOVA's requirement for normality, box-cox transformations were applied to non-normally distributed variables. The Scheffe test was applied to test for differences between least squares means derived from the fully adjusted models by food insecurity status, while accounting for multiple comparisons. Tests for trend assessed whether there was a linear relationship between group mean intakes and the severity of household food insecurity (as measured by the four-level categorical variable) in the fully adjusted models.

To ensure population-representativeness, survey weights were applied in all analyses, using a weighted bootstrap technique to estimate variance. A $P$-value of 0.05 was considered significant. Statistical analyses were performed using SAS 9.4 software (2013, SAS Institute Inc.).

\section{Results}

The prevalence of household food insecurity in this sample was $13.8 \%$, with $4.4 \%$ of individuals living in marginally food-insecure households, $6.7 \%$ in moderately foodinsecure households and $2.7 \%$ in severely food-insecure households. The distribution of household food insecurity status differed across age/sex groups, with a lower prevalence of food security among children than adults (Fig. 1). The distributions of household education and income differed by household food security status among all age/sex groups, with lower educational attainment among food-insecure households and an increasing proportion of households in the lowest income quintile as severity of food insecurity worsened (Table 2).

\section{Food selection and overall diet quality}

Food insecurity status was strongly related to HEI-C scores, the mean \% energy from ultra-processed foods and fruit and vegetable intakes across most groups when only age and sex were taken into account (Table 3). After adjustment for socio-demographic characteristics, there was no significant trend in HEI-C scores or fruit and vegetable intakes for any group, but moderate food insecurity was associated with significantly lower HEI-C scores than food security for children 1-8 years, men and women. Additionally, fruit and vegetable intakes were lower among younger children in moderately food-insecure households compared with the food secure and among women in severely food-insecure compared with moderately insecure households (Table 3). Among all four age/sex groups, the mean \% energy from ultra-processed foods was significantly higher among those with more severe food insecurity, with significant trends for all groups (Table 3). The difference between the mean \% energy from ultra-processed foods among food-secure and severely food-insecure younger children, men and women was approximately 10 percentage points.

\section{Macronutrient, sugar, sodium and fibre intakes}

There was no statistically significant difference in the mean proportion of energy from fat, carbohydrate, sugar or saturated fat by food insecurity status for any group (Table 4). The only difference observed in Na intakes per $1000 \mathrm{kcal}$

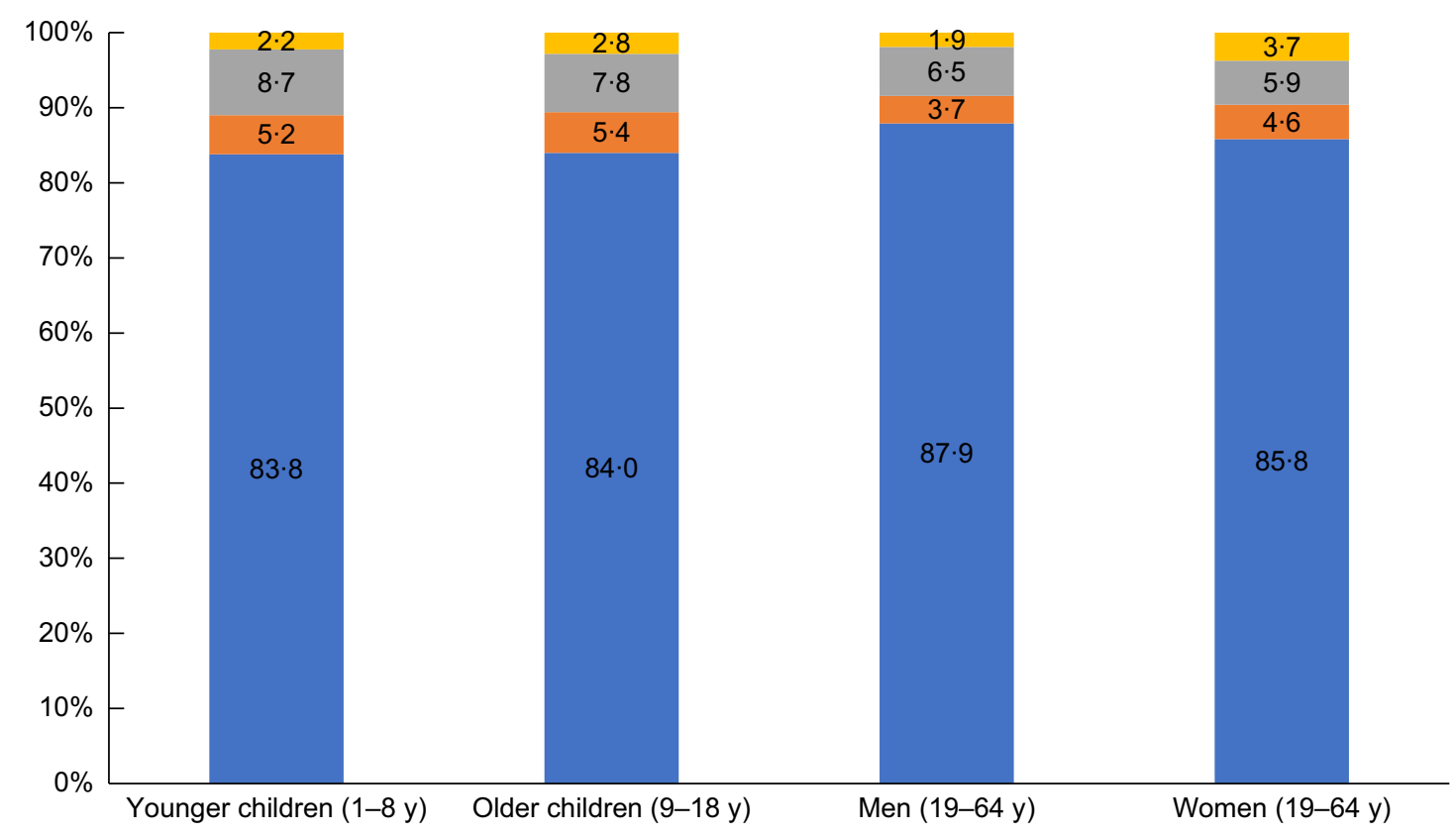

Fig. 1 (colour online) Prevalence of household food insecurity. $\sqsubset$, Food secure; $\sqsubset$, Marginal food insecurity; $\square$, Moderate food insecurity; $\sqsubset$, Severe food security 
Table 2 Household characteristics by age/sex and household food security status

\begin{tabular}{|c|c|c|c|c|c|c|c|c|c|}
\hline & \multicolumn{2}{|c|}{ Food-secure } & \multicolumn{2}{|c|}{$\begin{array}{l}\text { Marginally food } \\
\text { insecure }\end{array}$} & \multicolumn{2}{|c|}{$\begin{array}{l}\text { Moderately } \\
\text { food insecure }\end{array}$} & \multicolumn{2}{|c|}{$\begin{array}{l}\text { Severely food } \\
\text { insecure }\end{array}$} & \multirow[b]{2}{*}{$P>\chi^{2}$} \\
\hline & $\%$ & SE & $\%$ & SE & $\%$ & SE & $\%$ & SE & \\
\hline \multicolumn{10}{|l|}{ Younger Children, $1-8$ years } \\
\hline \multicolumn{10}{|l|}{ Household education } \\
\hline High school or below & $9 \cdot 1$ & 1.01 & $31 \cdot 3$ & 8.69 & 36.9 & 5.38 & $47 \cdot 2$ & $8 \cdot 17$ & \multirow[t]{3}{*}{$<0.001$} \\
\hline Some postsecondary* & 39.4 & 1.82 & 33.1 & $7 \cdot 64$ & 43.2 & 5.58 & 39.2 & $8 \cdot 31$ & \\
\hline Bachelor's degree or higher & 51.4 & 1.95 & $35 \cdot 7$ & 8.70 & 19.9 & 4.61 & $13 \cdot 6$ & 5.53 & \\
\hline \multicolumn{10}{|l|}{ Income quintile } \\
\hline 1 & $14 \cdot 0$ & 1.31 & $50 \cdot 6$ & 8.77 & 59.9 & 5.44 & $70 \cdot 3$ & $7 \cdot 83$ & \multirow[t]{2}{*}{$<0.001$} \\
\hline 2 to 5 & $86 \cdot 0$ & 1.31 & $49 \cdot 4$ & 8.77 & $40 \cdot 1$ & 5.44 & $29 \cdot 7$ & $7 \cdot 83$ & \\
\hline \multicolumn{10}{|l|}{ Older Children, 9-18 years } \\
\hline \multicolumn{10}{|l|}{ Household education } \\
\hline High school or below & $15 \cdot 4$ & $1 \cdot 13$ & $25 \cdot 2$ & $5 \cdot 01$ & 36.9 & 3.98 & $36 \cdot 1$ & 6.43 & \multirow[t]{3}{*}{$<0.001$} \\
\hline Some postsecondary* & $38 \cdot 2$ & 1.41 & $46 \cdot 7$ & $5 \cdot 86$ & 38.6 & 3.99 & 58.5 & 6.68 & \\
\hline Bachelor's degree or higher & 46.5 & 1.63 & $28 \cdot 1$ & $5 \cdot 38$ & $24 \cdot 6$ & $3 \cdot 84$ & 5.5 & 2.58 & \\
\hline \multicolumn{10}{|l|}{ Income quintile } \\
\hline 1 & $13 \cdot 6$ & 1.03 & $39 \cdot 9$ & $5 \cdot 86$ & $55 \cdot 7$ & $4 \cdot 37$ & $76 \cdot 4$ & $5 \cdot 71$ & \multirow[t]{2}{*}{$<0.001$} \\
\hline 2 to 5 & 86.4 & 1.03 & $60 \cdot 1$ & 5.86 & $44 \cdot 3$ & 4.37 & 23.6 & $5 \cdot 71$ & \\
\hline \multicolumn{10}{|l|}{ Women, 19-64 years } \\
\hline \multicolumn{10}{|l|}{ Household education } \\
\hline High school or below & $16 \cdot 7$ & 1.09 & $20 \cdot 4$ & 4.93 & $39 \cdot 7$ & 4.54 & 40.5 & 7.03 & \multirow[t]{3}{*}{$<0.001$} \\
\hline Some postsecondary* & $37 \cdot 1$ & 1.44 & $55 \cdot 8$ & 6.52 & $42 \cdot 8$ & 4.93 & $43 \cdot 1$ & 8.51 & \\
\hline Bachelor's degree or higher & $46 \cdot 2$ & 1.55 & 23.8 & $5 \cdot 11$ & $17 \cdot 6$ & 4.39 & $16 \cdot 4$ & 5.45 & \\
\hline \multicolumn{10}{|l|}{ Income quintile } \\
\hline 1 & $11 \cdot 2$ & 0.88 & 31.4 & 6.08 & $45 \cdot 4$ & $4 \cdot 77$ & $76 \cdot 9$ & $5 \cdot 07$ & \multirow[t]{2}{*}{$<0.001$} \\
\hline 2 to 5 & $88 \cdot 8$ & 0.88 & $68 \cdot 6$ & 6.08 & $54 \cdot 6$ & $4 \cdot 77$ & $23 \cdot 1$ & 5.07 & \\
\hline \multicolumn{10}{|l|}{ Men, 19-64 years } \\
\hline \multicolumn{10}{|l|}{ Household education } \\
\hline High school or below & $16 \cdot 3$ & $1 \cdot 16$ & $30 \cdot 3$ & 6.40 & $37 \cdot 0$ & $4 \cdot 76$ & $58 \cdot 7$ & 9.03 & \multirow[t]{3}{*}{$<0.001$} \\
\hline Some postsecondary ${ }^{*}$ & 40.5 & 1.67 & $55 \cdot 0$ & 7.04 & $38 \cdot 1$ & 4.88 & $28 \cdot 8$ & $7 \cdot 72$ & \\
\hline Bachelor's degree or higher & 43.2 & 1.64 & $14 \cdot 7$ & 3.90 & 24.9 & 4.56 & 12.5 & 6.47 & \\
\hline \multicolumn{10}{|l|}{ Income quintile } \\
\hline 1 & $11 \cdot 3$ & 1.28 & $23 \cdot 8$ & $5 \cdot 84$ & $47 \cdot 3$ & $4 \cdot 87$ & $62 \cdot 2$ & $8 \cdot 62$ & \multirow[t]{2}{*}{$<0.001$} \\
\hline 2 to 5 & 88.7 & 1.28 & $76 \cdot 2$ & $5 \cdot 84$ & 52.7 & 4.87 & 37.8 & 8.62 & \\
\hline
\end{tabular}

*Trade certificate or diploma, college/CEGEP/other non-university certificate or diploma, university certificate or diploma below the bachelor's level, or not stated.

was among young children. Those in severely food insecure households had higher mean intakes, and the trend was significant $\left(P_{\text {trend }}=0 \cdot 018\right)$. However, in the fully adjusted ANOVA model, mean $\mathrm{Na}$ intakes did not differ significantly among younger children by household food insecurity status $(P=0.097)$.

Compared with the food secure, protein comprised a significantly lower proportion of energy among older children in severely food-insecure households and men in moderately food-insecure households, and there was a significant trend in the \% energy from protein for older children $\left(P_{\text {trend }}=0 \cdot 010\right)$ (Table 4$)$. Dietary fibre per $1000 \mathrm{kcal}$ differed by food insecurity status only among women, with a lower mean intake among those who were severely food insecure and a significant negative trend $\left(P_{\text {trend }}=0 \cdot 001\right)$.

\section{Micronutrient intakes}

Few statistically significant differences were observed in micronutrient intakes by household food security status among fully adjusted models across age-sex strata. No statistically significant trends in micronutrient intakes per $1000 \mathrm{kcal}$ were observed for younger children, but younger children in moderately insecure households had significantly lower mean intakes of vitamins $\mathrm{A}, \mathrm{D}, \mathrm{B}_{6}$, $\mathrm{B}_{12}$, Ca, $\mathrm{P}$ and $\mathrm{K}$ compared with the food secure and significantly lower mean intakes per $1000 \mathrm{kcal}$ of vitamin D, riboflavin, vitamin $\mathrm{B}_{12}, \mathrm{Ca}, \mathrm{Mg}$ and $\mathrm{P}$ than marginally food-insecure children (Table 5). The $\mathrm{Ca}$ and $\mathrm{P}$ intakes/ $1000 \mathrm{kcal}$ of younger children in marginally food-insecure households were, on average, significantly higher than children in food-secure households (Table 5).

Among older children, severe food insecurity was associated with significantly lower mean thiamin, niacin, $\mathrm{P}$ and $\mathrm{Zn}$ intakes $/ 1000 \mathrm{kcal}$, and there were significant trends for thiamin, niacin, $\mathrm{Mg}$ and $\mathrm{Zn}\left(P_{\text {trend }}=0.021,0 \cdot 044,0.039\right.$ and 0.006, respectively).

Among women, there was a significant trend for $\mathrm{Mg}$ $\left(P_{\text {trend }}=0.008\right)$, but no other significant differences in mean micronutrient intakes $/ 1000 \mathrm{kcal}$ by food insecurity status once socio-demographic characteristics were taken into account (Table 5).

Among men, there were significant trends in vitamin $\mathrm{C}$ and $\mathrm{Zn}$ intakes per $1000 \mathrm{kcal}$ by food insecurity status ( $P_{\text {trend }}=0.035$ and 0.017 , respectively) (Table 5). Additionally, men in moderately food-insecure households had significantly lower mean intakes of niacin, $\mathrm{Mg}, \mathrm{P}$ and 
Table 3 Indicators of diet quality by age/sex and household food insecurity status

\begin{tabular}{|c|c|c|c|c|c|c|c|c|c|c|c|}
\hline & \multicolumn{2}{|c|}{ Food-secure } & \multicolumn{2}{|c|}{$\begin{array}{l}\text { Marginally food } \\
\text { insecure }\end{array}$} & \multicolumn{2}{|c|}{$\begin{array}{l}\text { Moderately food } \\
\text { insecure }\end{array}$} & \multicolumn{2}{|c|}{$\begin{array}{l}\text { Severely food } \\
\text { insecure }\end{array}$} & \multirow{2}{*}{$\begin{array}{l}P \text {-valuet } \\
\text { (model 1) }\end{array}$} & \multirow{2}{*}{$\begin{array}{c}P \text {-value } \\
\text { (model 2) }\end{array}$} & \multirow{2}{*}{$\begin{array}{c}P_{\text {trend }} \\
\text { (model 2) }\end{array}$} \\
\hline & Mean* & SEM & Mean* & SEM & Mean* & SEM & Mean* & SEM & & & \\
\hline \multicolumn{12}{|c|}{ Healthy Eating Index - Canada 2005 score } \\
\hline Younger children, $2-8$ years $\$$ & $71.5^{\mathrm{a}}$ & 0.42 & $71 \cdot 5^{\mathrm{a}}$ & 1.80 & $65.0^{b}$ & 1.29 & $68 \cdot 0^{a, b}$ & 1.71 & $<0.001$ & 0.079 & 0.467 \\
\hline Older children, $9-18$ years & $62 \cdot 7^{\mathrm{a}}$ & 0.34 & $59 \cdot 9^{\mathrm{a}}$ & 1.36 & $60 \cdot 8^{\mathrm{a}}$ & 1.24 & $58 \cdot 6^{\mathrm{a}}$ & 1.87 & 0.014 & 0.149 & 0.159 \\
\hline Women, $19-64$ years & $62.9^{a}$ & 0.40 & $62 \cdot 2^{a, b}$ & 1.95 & $57 \cdot 8^{\mathrm{b}}$ & 1.27 & $57 \cdot 7^{a, b}$ & 1.83 & $<0.001$ & 0.027 & 0.061 \\
\hline Men, $19-64$ years & $60 \cdot 4^{\mathrm{a}}$ & 0.43 & $56 \cdot 8^{\mathrm{a}}$ & $2 \cdot 15$ & $54.7^{\mathrm{b}}$ & 1.31 & $56 \cdot 8^{\mathrm{a}, \mathrm{b}}$ & $2 \cdot 40$ & $<0.001$ & 0.008 & 0.196 \\
\hline \multicolumn{12}{|l|}{ Fruit \& vegetables (servings) } \\
\hline Younger children, $1-8$ years & $4 \cdot 0^{\mathrm{a}}$ & $0 \cdot 10$ & $3 \cdot 4^{a, b}$ & 0.31 & $2.9^{b}$ & 0.20 & $3 \cdot 5^{\mathrm{a}, \mathrm{b}}$ & 0.56 & 0.001 & 0.148 & 0.436 \\
\hline Older children, $9-18$ years & $4 \cdot 1^{\mathrm{a}}$ & 0.09 & $4 \cdot 4^{\mathrm{a}}$ & 0.49 & $3.6^{a}$ & 0.28 & $4 \cdot 3^{\mathrm{a}}$ & 0.57 & 0.207 & 0.562 & 0.968 \\
\hline Women, $19-64$ years & $4 \cdot 4^{\mathrm{a}, \mathrm{b}}$ & $0 \cdot 10$ & $3 \cdot 8^{a, b}$ & 0.40 & $4 \cdot 0^{\mathrm{a}}$ & 0.30 & $3.0^{\mathrm{b}}$ & 0.53 & 0.007 & 0.223 & 0.106 \\
\hline Men, $19-64$ years & $4 \cdot 6^{\mathrm{a}}$ & 0.10 & $4 \cdot 1^{\mathrm{a}}$ & 0.48 & $3.8^{\mathrm{a}}$ & 0.31 & $3.7^{a}$ & 0.63 & 0.004 & 0.510 & 0.408 \\
\hline \multicolumn{12}{|l|}{ Ultra-processed intake (\% energy) } \\
\hline Younger children, $1-8$ years & $41 \cdot 6^{a}$ & 0.59 & $40 \cdot 6^{a}$ & 3.46 & $50 \cdot 2^{b}$ & $1 \cdot 88$ & $52 \cdot 0^{\mathrm{b}, \mathrm{c}}$ & $2 \cdot 77$ & $<0.001$ & 0.005 & 0.002 \\
\hline Older children, $9-18$ years & $46 \cdot 8^{a}$ & 0.54 & $49 \cdot 7^{a, b}$ & 1.89 & $47 \cdot 3^{a, b}$ & 1.62 & $47 \cdot 3^{\mathrm{b}}$ & 1.62 & 0.073 & 0.062 & 0.049 \\
\hline Women, $19-64$ years & $37 \cdot 6^{a}$ & 0.56 & $38.9^{a}$ & 1.98 & $40 \cdot 9^{a}$ & 1.69 & $45 \cdot 8^{\mathrm{b}}$ & $2 \cdot 40$ & 0.002 & 0.029 & 0.003 \\
\hline Men, $19-64$ years & $37.5^{\mathrm{a}}$ & 0.66 & $42 \cdot 5^{a, b}$ & 2.87 & $46.7^{b}$ & 2.37 & $47.0^{\mathrm{b}}$ & 3.70 & $<0.001$ & 0.002 & 0.009 \\
\hline
\end{tabular}

SEM, standard error of the mean.

*Separate models were run for each age-sex stratum. Means were derived from ANOVA, adjusted for age and sex (children's models only).

$\lceil P$-values were derived from ANOVA, adjusted for age and sex (children's models only) with household food insecurity status entered as a four-level categorical variable.

$\ddagger P$-values were derived from ANOVA, adjusted for age, sex (children's models only), education, income and region, with household food insecurity status entered as a four-level categorical variable.

§Among children aged 2-8 years as Canada's Food Guide 2007 recommendations were created for $\geq 2$ years.

a,b,c Mean values within a row with unlike superscript letters were significantly different $(P<0.05)$. 
Table 4 Macronutrient, fibre, sugar and sodium intakes by age/sex and household food insecurity status

\begin{tabular}{|c|c|c|c|c|c|c|c|c|c|c|c|}
\hline & \multicolumn{2}{|c|}{ Food-secure } & \multicolumn{2}{|c|}{$\begin{array}{l}\text { Marginally food } \\
\text { insecure }\end{array}$} & \multicolumn{2}{|c|}{$\begin{array}{l}\text { Moderately food } \\
\text { insecure }\end{array}$} & \multicolumn{2}{|c|}{$\begin{array}{l}\text { Severely food } \\
\text { insecure }\end{array}$} & \multirow{2}{*}{$\begin{array}{l}P \text {-value } \dagger \\
\text { (model 1) }\end{array}$} & \multirow{2}{*}{$\begin{array}{c}P \text {-value } \\
\text { (model 2) }\end{array}$} & \multirow{2}{*}{$\begin{array}{c}P_{\text {trend }} \ddagger \\
\text { (model 2) }\end{array}$} \\
\hline & Mean* & SEM & Mean* & SEM & Mean* & SEM & Mean* & SEM & & & \\
\hline \multicolumn{12}{|l|}{ Protein (\% energy) } \\
\hline Younger children, $1-8$ years & $16 \cdot 0^{\mathrm{a}}$ & $0 \cdot 16$ & $15 \cdot 3^{\mathrm{a}}$ & 0.44 & $15 \cdot 0^{\mathrm{a}}$ & 0.39 & $15 \cdot 2^{\mathrm{a}}$ & 0.56 & 0.039 & 0.338 & 0.493 \\
\hline Older children, $9-18$ years & $16 \cdot 0^{\mathrm{a}}$ & 0.18 & $15 \cdot 0^{\mathrm{a}, \mathrm{b}}$ & 0.50 & $15 \cdot 5^{\mathrm{a}}$ & 0.39 & $13 \cdot 8^{\mathrm{b}}$ & 0.50 & 0.001 & 0.027 & 0.010 \\
\hline Women, $19-64$ years & $17 \cdot 3^{\mathrm{a}}$ & 0.17 & $17 \cdot 7^{\mathrm{a}}$ & 0.81 & $17 \cdot 3^{\mathrm{a}}$ & 0.69 & $16 \cdot 5^{a}$ & 0.71 & 0.689 & 0.786 & 0.407 \\
\hline Men19-64 years & $17 \cdot 7^{\mathrm{a}}$ & 0.17 & $16 \cdot 4^{a, b}$ & 0.57 & $16 \cdot 1^{\mathrm{b}}$ & 0.61 & $16 \cdot 6^{a, b}$ & 0.88 & 0.011 & 0.015 & 0.167 \\
\hline \multicolumn{12}{|l|}{ Fat (\% energy) } \\
\hline Younger children, $1-8$ years & $31 \cdot 0^{\mathrm{a}}$ & 0.25 & $30 \cdot 1^{a}$ & 1.03 & $31.9^{a}$ & 0.97 & $31.9^{a}$ & 0.97 & 0.444 & 0.279 & 0.073 \\
\hline Older children, $9-18$ years & $31 \cdot 7^{a}$ & 0.24 & $32 \cdot 7^{\mathrm{a}}$ & 1.11 & $31 \cdot 5^{a}$ & 0.86 & $33 \cdot 1^{\mathrm{a}}$ & 1.20 & 0.502 & 0.297 & 0.268 \\
\hline Women, $19-64$ years & $32.9^{a}$ & 0.31 & $32 \cdot 7^{\mathrm{a}}$ & 1.05 & $32 \cdot 8^{\mathrm{a}}$ & 0.93 & $31.0^{\mathrm{a}}$ & $1 \cdot 18$ & 0.445 & 0.737 & 0.357 \\
\hline Men, $19-64$ years & $32 \cdot 7^{a}$ & 0.29 & $34 \cdot 2^{\mathrm{a}}$ & 1.68 & $32 \cdot 1^{\mathrm{a}}$ & $1 \cdot 18$ & $32 \cdot 4^{\mathrm{a}}$ & 1.53 & 0.787 & 0.772 & 0.607 \\
\hline \multicolumn{12}{|l|}{ Carbohydrates (\% energy) } \\
\hline Younger children, $1-8$ years & $54 \cdot 4^{\mathrm{a}}$ & 0.35 & $55 \cdot 9^{a}$ & $1 \cdot 18$ & $54 \cdot 3^{a}$ & $1 \cdot 17$ & $53 \cdot 6^{a}$ & $1 \cdot 30$ & 0.608 & 0.567 & 0.247 \\
\hline Older children, $9-18$ years & $53 \cdot 4^{\mathrm{a}}$ & 0.31 & $53 \cdot 4^{\mathrm{a}}$ & $1 \cdot 16$ & $54 \cdot 2^{\mathrm{a}}$ & 0.88 & $54 \cdot 3^{\mathrm{a}}$ & 1.39 & 0.804 & 0.965 & 0.789 \\
\hline Women, $19-64$ years & $48 \cdot 8^{\mathrm{a}}$ & 0.36 & $49 \cdot 7^{\mathrm{a}}$ & 1.34 & $50 \cdot 0^{\mathrm{a}}$ & 1.35 & $51 \cdot 8^{\mathrm{a}}$ & 1.93 & 0.312 & 0.681 & 0.319 \\
\hline Men, $19-64$ years & $47 \cdot 2^{\mathrm{a}}$ & 0.35 & $47 \cdot 9^{\mathrm{a}}$ & 1.97 & $48 \cdot 5^{\mathrm{a}}$ & 1.23 & $49 \cdot 3^{a}$ & 2.01 & 0.555 & 0.960 & 0.646 \\
\hline \multicolumn{12}{|l|}{ Sugar (\% energy) } \\
\hline Younger children, $1-8$ years & $25 \cdot 7^{a}$ & 0.30 & $24.9^{\mathrm{a}}$ & 1.90 & $24 \cdot 6^{\mathrm{a}}$ & 1.01 & $25 \cdot 8^{\mathrm{a}}$ & 1.33 & 0.700 & 0.443 & 0.704 \\
\hline Older children, $9-18$ years & $23 \cdot 0^{a}$ & 0.27 & $22 \cdot 8^{\mathrm{a}}$ & 1.30 & $22 \cdot 8^{\mathrm{a}}$ & 0.79 & $23 \cdot 7^{a}$ & 1.46 & 0.955 & 0.482 & 0.163 \\
\hline Women, $19-64$ years & $19 \cdot 2^{\mathrm{a}}$ & 0.29 & $19 \cdot 4^{\mathrm{a}}$ & 0.92 & $21 \cdot 1^{\mathrm{a}}$ & 1.03 & $22 \cdot 0^{\mathrm{a}}$ & 1.41 & 0.097 & 0.194 & 0.062 \\
\hline Men, $19-64$ years & $17 \cdot 9^{\mathrm{a}}$ & 0.25 & $19 \cdot 6^{\mathrm{a}}$ & 1.33 & $19 \cdot 5^{\mathrm{a}}$ & $1 \cdot 26$ & $19 \cdot 4^{\mathrm{a}}$ & 1.78 & 0.269 & 0.171 & 0.285 \\
\hline \multicolumn{12}{|l|}{ Fibre (g/1000 kcal) } \\
\hline Younger children, $1-8$ years & $9 \cdot 0^{\mathrm{a}}$ & 0.14 & $8 \cdot 4^{\mathrm{a}}$ & 0.49 & $8.0^{\mathrm{a}}$ & 0.37 & $8 \cdot 8^{\mathrm{a}}$ & 0.42 & 0.063 & 0.382 & 0.326 \\
\hline Older children, $9-18$ years & $8.4^{\mathrm{a}}$ & 0.09 & $8 \cdot 4^{\mathrm{a}}$ & 0.09 & $8 \cdot 2^{\mathrm{a}}$ & 0.56 & $7 \cdot 7^{\mathrm{a}}$ & 0.39 & 0.211 & 0.323 & 0.112 \\
\hline Women, $19-64$ years & $10 \cdot 6^{a}$ & 0.16 & $9 \cdot 3^{a, b}$ & 0.49 & $9 \cdot 2^{a, b}$ & 0.43 & $8 \cdot 0^{b}$ & 0.51 & $<0.001$ & 0.002 & 0.001 \\
\hline Men, 19-64 years & $8 \cdot 8^{a}$ & 0.13 & $9 \cdot 0^{\mathrm{a}}$ & 0.63 & $8 \cdot 1^{a}$ & 0.44 & $7 \cdot 7^{\mathrm{a}}$ & 0.53 & 0.055 & 0.245 & 0.130 \\
\hline \multicolumn{12}{|l|}{ Saturated fat (\% energy) } \\
\hline Younger children, $1-8$ years & $11 \cdot 8^{a}$ & 0.14 & $11.9^{a}$ & 0.71 & $11.9^{a}$ & 0.44 & $12 \cdot 0^{\mathrm{a}}$ & 0.69 & 0.987 & 0.947 & 0.669 \\
\hline Older children, $9-18$ years & $11 \cdot 1^{\mathrm{a}}$ & 0.11 & $10 \cdot 7^{a}$ & 0.48 & $10 \cdot 9^{a}$ & 0.39 & $10 \cdot 7^{a}$ & 0.50 & 0.602 & 0.974 & 0.901 \\
\hline Women, $19-64$ years & $10 \cdot 6^{a}$ & 0.13 & $10 \cdot 7^{a}$ & 0.44 & $10 \cdot 7^{a}$ & 0.39 & $9 \cdot 6^{a}$ & 0.58 & 0.332 & 0.496 & 0.201 \\
\hline Men, 19-64 years & $10 \cdot 3^{a}$ & 0.11 & $11 \cdot 4^{\mathrm{a}}$ & 0.82 & $10 \cdot 6^{a}$ & 0.48 & $9 \cdot 8^{\mathrm{a}}$ & 0.56 & 0.429 & 0.413 & 0.182 \\
\hline \multicolumn{12}{|l|}{$\mathrm{Na}(\mathrm{mg} / 1000 \mathrm{kcal})$} \\
\hline Younger children, 1-8 years & $1299 \cdot 1^{a}$ & 14.54 & $1329 \cdot 8^{a, b}$ & 73.33 & $1403 \cdot 1^{\mathrm{a}, \mathrm{b}}$ & 63.38 & $1522 \cdot 7^{b}$ & 106.96 & 0.042 & 0.097 & 0.018 \\
\hline Older children, $9-18$ years & $1452 \cdot 7^{\mathrm{a}}$ & 13.88 & $1515 \cdot 4^{\mathrm{a}}$ & $50 \cdot 42$ & $1434 \cdot 2^{\mathrm{a}}$ & 44.72 & $1479 \cdot 5^{a}$ & 74.59 & 0.572 & 0.591 & 0.951 \\
\hline Women, $19-64$ years & $1482 \cdot 9^{\mathrm{a}}$ & 18.75 & $1613 \cdot 8^{\mathrm{a}}$ & $147 \cdot 37$ & $1458 \cdot 5^{\mathrm{a}}$ & $45 \cdot 11$ & $1403 \cdot 3^{a}$ & 53.39 & 0.591 & 0.769 & 0.405 \\
\hline Men, $19-64$ years & $1480 \cdot 1^{a}$ & $16 \cdot 41$ & $1605 \cdot 4^{\mathrm{a}}$ & $116 \cdot 26$ & $1465 \cdot 9^{a}$ & 63.31 & $1603 \cdot 3^{a}$ & 180.79 & 0.765 & 0.740 & 0.802 \\
\hline
\end{tabular}

SEM, standard error of the mean.

*Separate models were run for each age-sex stratum. Means were derived from ANOVA, adjusted for age and sex (children's models only).

(ched from ANOVA, adjusted for age and sex (children's models only) with household food insecurity status entered as a four-level categorical variable.

$¥ P$-values were derived from ANOVA, adjusted for age, sex (children's models only), education, income and region, with household food insecurity status entered as a 4-level categorical variable.

a,b,c Mean values within a row with unlike superscript letters were significantly different $(P<0.05)$. 
Table 5 Micronutrient intakes per $1000 \mathrm{kcal}$ by age/sex and household food insecurity status

\begin{tabular}{|c|c|c|c|c|c|c|c|c|c|c|c|}
\hline & \multicolumn{2}{|c|}{ Food-secure } & \multicolumn{2}{|c|}{$\begin{array}{l}\text { Marginally food } \\
\text { insecure }\end{array}$} & \multicolumn{2}{|c|}{$\begin{array}{c}\text { Moderately food } \\
\text { insecure }\end{array}$} & \multicolumn{2}{|c|}{$\begin{array}{l}\text { Severely food } \\
\text { insecure }\end{array}$} & \multirow[b]{2}{*}{$P$-value† (model 1) } & \multirow[b]{2}{*}{$P$-value $\neq$ (model 2) } & \multirow[b]{2}{*}{$P_{\text {trend }}($ model 2$)$} \\
\hline & Mean* $^{*}$ & SEM & Mean* $^{*}$ & SEM & Mean* $^{*}$ & SEM & Mean* & SEM & & & \\
\hline \multicolumn{12}{|l|}{ Vitamin A (RAE/1000 kcal) } \\
\hline Younger children, $1-8$ years & $401 \cdot 3^{\mathrm{a}}$ & 9.60 & $339 \cdot 6^{a, b}$ & $27 \cdot 27$ & $342.9^{b}$ & 21.88 & $371 \cdot 2^{a, b}$ & $28 \cdot 80$ & 0.007 & $0 \cdot 106$ & 0.757 \\
\hline Older children, $9-18$ years & $333 \cdot 6^{\mathrm{a}}$ & $10 \cdot 61$ & $319 \cdot 6^{a}$ & $30 \cdot 13$ & $294 \cdot 6^{\mathrm{a}}$ & $18 \cdot 30$ & $254 \cdot 6^{a}$ & $19 \cdot 40$ & 0.004 & 0.478 & $0 \cdot 124$ \\
\hline Women, $19-64$ years & $401 \cdot 6^{\mathrm{a}}$ & $15 \cdot 00$ & $372 \cdot 9^{\mathrm{a}}$ & 31.23 & $354 \cdot 0^{\mathrm{a}}$ & 37.89 & $336 \cdot 4^{\mathrm{a}}$ & 47.49 & 0.094 & 0.855 & 0.381 \\
\hline Men, 19-64 years & $335 \cdot 3^{a, b}$ & 11.45 & $356 \cdot 8^{\mathrm{a}}$ & $40 \cdot 86$ & $277 \cdot 8^{\mathrm{b}}$ & 27.58 & $298 \cdot 4^{a, b}$ & $47 \cdot 75$ & 0.063 & 0.192 & 0.164 \\
\hline \multicolumn{12}{|l|}{ Vitamin C (mg/1000 kcal) } \\
\hline Younger children, 1-8 years & $76 \cdot 1^{\mathrm{a}}$ & 2.09 & $63 \cdot 1^{\mathrm{a}}$ & 5.04 & $70 \cdot 0^{\mathrm{a}}$ & $6 \cdot 88$ & $78 \cdot 2^{\mathrm{a}}$ & $12 \cdot 63$ & 0.541 & 0.733 & 0.962 \\
\hline Older children, 9-18 years & $60 \cdot 6^{a}$ & 1.54 & $60 \cdot 9^{a}$ & 5.94 & $55 \cdot 7^{\mathrm{a}}$ & 4.45 & $67 \cdot 6^{\mathrm{a}}$ & $7 \cdot 21$ & 0.161 & 0.221 & 0.597 \\
\hline Women, 19-64 years & $64 \cdot 3^{\mathrm{a}}$ & 2.57 & $63 \cdot 3^{\mathrm{a}}$ & $6 \cdot 82$ & $58 \cdot 8^{\mathrm{a}}$ & $7 \cdot 32$ & $47 \cdot 5^{\mathrm{a}}$ & $6 \cdot 12$ & 0.046 & 0.354 & 0.331 \\
\hline Men, 19-64 years & $50 \cdot 1^{\mathrm{a}, \mathrm{c}}$ & 1.61 & $49 \cdot 0^{a, b, c}$ & $10 \cdot 82$ & $49 \cdot 5^{\mathrm{a}, \mathrm{b}}$ & 7.92 & $29 \cdot 6^{\mathrm{b}}$ & 4.89 & 0.005 & 0.089 & 0.035 \\
\hline \multicolumn{12}{|l|}{ Vitamin D (mcg/1000 kcal) } \\
\hline Younger children, $1-8$ years & $4 \cdot 1^{\mathrm{a}}$ & 0.09 & $4.9^{\mathrm{a}}$ & 0.47 & $3 \cdot 2^{b}$ & 0.22 & $3 \cdot 8^{a, b}$ & 0.30 & 0.006 & 0.009 & 0.062 \\
\hline Older children, 9-18 years & $2 \cdot 9^{\mathrm{a}}$ & 0.08 & $2 \cdot 4^{\mathrm{a}}$ & 0.22 & $2 \cdot 6^{\mathrm{a}}$ & 0.13 & $2 \cdot 2^{\mathrm{a}}$ & 0.20 & 0.077 & 0.343 & 0.503 \\
\hline Women, 19-64 years & $2 \cdot 6^{\mathrm{a}}$ & 0.07 & $2 \cdot 7^{\mathrm{a}}$ & 0.23 & $3.0^{\mathrm{a}}$ & 0.32 & $2 \cdot 3^{a}$ & 0.21 & 0.473 & 0.474 & 0.428 \\
\hline Men, 19-64 years & $2 \cdot 4^{\mathrm{a}}$ & 0.08 & $2 \cdot 6^{a}$ & 0.50 & $2 \cdot 1^{\mathrm{a}}$ & 0.18 & $2 \cdot 3^{\mathrm{a}}$ & 0.45 & 0.379 & 0.553 & 0.259 \\
\hline \multicolumn{12}{|l|}{ Thiamin (mg/1000 kcal) } \\
\hline Younger children, $1-8$ years & $0.9^{a}$ & 0.01 & $0.9^{\mathrm{a}}$ & 0.08 & $0.9^{\mathrm{a}}$ & 0.03 & $0.9^{a}$ & 0.04 & 0.595 & 0.498 & 0.480 \\
\hline Older children, $9-18$ years & $0.9^{a}$ & 0.01 & $0.9^{a, b}$ & 0.06 & $0.9^{a, b}$ & 0.04 & $0 \cdot 8^{b}$ & 0.04 & 0.072 & $0 \cdot 131$ & 0.021 \\
\hline Women, $19-64$ years & $0.9^{\mathrm{a}}$ & 0.01 & $0.9^{\mathrm{a}}$ & 0.04 & $0.9^{\mathrm{a}}$ & 0.03 & $0.8^{\mathrm{a}}$ & 0.04 & 0.881 & 0.715 & 0.739 \\
\hline Men, 19-64 years & $0 \cdot 8^{\mathrm{a}}$ & 0.01 & $0.9^{a}$ & 0.04 & $0 \cdot 8^{\mathrm{a}}$ & 0.04 & $0.9^{a}$ & 0.07 & 0.635 & 0.594 & 0.686 \\
\hline \multicolumn{12}{|l|}{ Riboflavin (mg/1000 kcal) } \\
\hline Younger children, 1-8 years & $1 \cdot 2^{\mathrm{a}}$ & 0.01 & $1 \cdot 3^{\mathrm{a}}$ & 0.07 & $1 \cdot 1^{b}$ & 0.03 & $1 \cdot 1^{\mathrm{a}, \mathrm{b}}$ & 0.05 & 0.008 & 0.034 & 0.081 \\
\hline Older children, $9-18$ years & $1.0^{\mathrm{a}}$ & 0.01 & $0.9^{b}$ & 0.04 & $0.9^{a, b}$ & 0.02 & $0.9^{a, b}$ & 0.05 & 0.004 & 0.089 & 0.213 \\
\hline Women, 19-64 years & $1 \cdot 1^{\mathrm{a}}$ & 0.01 & $1 \cdot 1^{\mathrm{a}}$ & 0.05 & $1 \cdot 1^{\mathrm{a}}$ & 0.04 & $1 \cdot 1^{\mathrm{a}}$ & 0.05 & 0.669 & 0.631 & 0.807 \\
\hline Men, 19-64 years & $1 \cdot 0^{\mathrm{a}}$ & 0.01 & $1 \cdot 0^{\mathrm{a}}$ & 0.05 & $1 \cdot 0^{\mathrm{a}}$ & 0.05 & $1.0^{\mathrm{a}}$ & 0.07 & 0.052 & 0.479 & 0.778 \\
\hline \multicolumn{12}{|l|}{ Niacin (mg/1000 kcal) } \\
\hline Younger children, $1-8$ years & $17 \cdot 9^{\mathrm{a}}$ & 0.19 & $17 \cdot 0^{\mathrm{a}}$ & 0.81 & $17 \cdot 4^{\mathrm{a}}$ & 0.55 & $16 \cdot 9^{\mathrm{a}}$ & 0.63 & 0.338 & 0.856 & 0.879 \\
\hline Older children, 9-18 years & $19 \cdot 1^{\mathrm{a}}$ & 0.24 & $18 \cdot 0^{\mathrm{a}, \mathrm{b}}$ & 0.68 & $18 \cdot 6^{\mathrm{a}, \mathrm{b}}$ & 0.54 & $17 \cdot 1^{\mathrm{b}}$ & 0.61 & 0.065 & 0.143 & 0.044 \\
\hline Women, 19-64 years & $21 \cdot 2^{\mathrm{a}}$ & 0.25 & $21 \cdot 7^{a}$ & $1 \cdot 18$ & $22 \cdot 3^{\mathrm{a}}$ & 0.95 & $21 \cdot 1^{\mathrm{a}}$ & 0.85 & 0.602 & 0.575 & 0.771 \\
\hline Men, 19-64 years & $22 \cdot 1^{\mathrm{a}}$ & 0.26 & $21 \cdot 0^{a, b}$ & 0.99 & $20 \cdot 3^{b}$ & 0.92 & $21 \cdot 1^{a, b}$ & 0.96 & 0.027 & 0.097 & 0.537 \\
\hline \multicolumn{12}{|l|}{ Vitamin $B_{6}(\mathrm{mg} / 1000 \mathrm{kcal})$} \\
\hline Younger children, $1-8$ years & $0.8^{\mathrm{a}}$ & 0.01 & $0.9^{a, b}$ & 0.09 & $0.7^{b}$ & 0.03 & $0.8^{\mathrm{a}, \mathrm{b}}$ & 0.05 & 0.004 & 0.017 & 0.084 \\
\hline Older children, $9-18$ years & $0 \cdot 8^{a}$ & 0.01 & $0 \cdot 8^{\mathrm{a}}$ & 0.04 & $0 \cdot 8^{\mathrm{a}}$ & 0.05 & $0 \cdot 7^{\mathrm{a}}$ & 0.04 & 0.325 & 0.356 & 0.083 \\
\hline Women, 19-64 years & $1 \cdot 0^{\mathrm{a}}$ & 0.01 & $0.9^{\mathrm{a}}$ & 0.05 & $1 \cdot 0^{\mathrm{a}}$ & 0.05 & $0.9^{\mathrm{a}}$ & 0.05 & 0.801 & 0.869 & 0.883 \\
\hline \multirow{2}{*}{\multicolumn{12}{|c|}{ Folate (DFE/1000 kcal) }} \\
\hline & & & & & & & & & & & \\
\hline Younger children, 1-8 years & $226 \cdot 4^{\mathrm{a}}$ & 3.13 & $249 \cdot 6^{\mathrm{a}}$ & 23.31 & $224 \cdot 1^{\mathrm{a}}$ & $9 \cdot 61$ & $216 \cdot 7^{\mathrm{a}}$ & $15 \cdot 78$ & 0.716 & 0.543 & 0.811 \\
\hline Older children, $9-18$ years & $239 \cdot 9^{a}$ & $2 \cdot 91$ & $236 \cdot 5^{a}$ & 11.58 & $232 \cdot 1^{\mathrm{a}}$ & $9 \cdot 16$ & $218 \cdot 6^{a}$ & $10 \cdot 89$ & 0.366 & 0.454 & 0.155 \\
\hline Women, 19-64 years & $252 \cdot 8^{\mathrm{a}}$ & 3.74 & $247 \cdot 6^{a}$ & $13 \cdot 78$ & $230 \cdot 2^{\mathrm{a}}$ & $8 \cdot 66$ & $228 \cdot 9^{\mathrm{a}}$ & $12 \cdot 53$ & 0.028 & 0.678 & 0.361 \\
\hline Men, 19-64 years & $237.4^{\mathrm{a}}$ & 2.93 & $218 \cdot 9^{\mathrm{a}}$ & $12 \cdot 20$ & $218 \cdot 2^{\mathrm{a}}$ & $10 \cdot 48$ & $228 \cdot 3^{\mathrm{a}}$ & $15 \cdot 91$ & 0.088 & 0.252 & 0.664 \\
\hline \multicolumn{12}{|l|}{ Vitamin $B_{12}(\mathrm{mcg} / 1000 \mathrm{kcal})$} \\
\hline Younger children, $1-8$ years & $2 \cdot 4^{\mathrm{a}}$ & 0.04 & $2 \cdot 5^{\mathrm{a}}$ & $0 \cdot 18$ & $2 \cdot 1^{b}$ & 0.13 & $2 \cdot 2^{\mathrm{a}, \mathrm{b}}$ & $0 \cdot 16$ & 0.087 & $0 \cdot 157$ & 0.174 \\
\hline Older children, $9-18$ years & $2 \cdot 2^{\mathrm{a}}$ & 0.11 & $1 \cdot 7^{\mathrm{a}}$ & 0.14 & $1.9^{\mathrm{a}}$ & 0.09 & $1.7^{\mathrm{a}}$ & $0 \cdot 16$ & 0.009 & 0.078 & 0.211 \\
\hline Women, $19-64$ years & $2 \cdot 2^{a}$ & 0.08 & $2 \cdot 3^{\mathrm{a}}$ & 0.22 & $2 \cdot 4^{\mathrm{a}}$ & 0.32 & $2 \cdot 6^{\mathrm{a}}$ & 0.44 & 0.390 & 0.653 & 0.385 \\
\hline
\end{tabular}




\section{Public Health Nutrition}

Table 5 Continued

\begin{tabular}{|c|c|c|c|c|c|c|c|c|c|c|c|}
\hline & \multicolumn{2}{|c|}{ Food-secure } & \multicolumn{2}{|c|}{$\begin{array}{l}\text { Marginally food } \\
\text { insecure }\end{array}$} & \multicolumn{2}{|c|}{$\begin{array}{l}\text { Moderately food } \\
\text { insecure }\end{array}$} & \multicolumn{2}{|c|}{$\begin{array}{l}\text { Severely food } \\
\text { insecure }\end{array}$} & \multirow[b]{2}{*}{$P$-value† (model 1) } & \multirow[b]{2}{*}{$P$-value $\ddagger$ (model 2) } & \multirow[b]{2}{*}{$P_{\text {trend }}($ model 2$)$} \\
\hline & Mean* & SEM & Mean* & SEM & Mean* & SEM & Mean* & SEM & & & \\
\hline Men, 19-64 years & $2 \cdot 2^{\mathrm{a}}$ & 0.06 & $2 \cdot 1^{a}$ & 0.19 & $2 \cdot 1^{a}$ & 0.11 & $2 \cdot 1^{a}$ & 0.29 & 0.928 & 0.895 & 0.527 \\
\hline \multicolumn{12}{|l|}{ Calcium (mg/1000 kcal) } \\
\hline Younger children, $1-8$ years & $650 \cdot 4^{\mathrm{a}}$ & 8.85 & $727 \cdot 4^{b}$ & 43.44 & $573 \cdot 9^{c}$ & $24 \cdot 32$ & $621 \cdot 8^{a, b, c}$ & $40 \cdot 82$ & 0.005 & 0.015 & $0 \cdot 240$ \\
\hline Older children, $9-18$ years & $492 \cdot 8^{a}$ & $6 \cdot 34$ & $430 \cdot 9^{\mathrm{a}}$ & 22.51 & $452 \cdot 0^{\mathrm{a}}$ & $16 \cdot 70$ & $422 \cdot 5^{\mathrm{a}}$ & 24.27 & 0.003 & 0.423 & 0.595 \\
\hline Women, $19-64$ years & $450 \cdot 0^{\mathrm{a}}$ & $6 \cdot 41$ & $459.9^{\mathrm{a}}$ & 24.01 & $418 \cdot 2^{\mathrm{a}}$ & $21 \cdot 26$ & $442 \cdot 3^{\mathrm{a}}$ & $27 \cdot 41$ & 0.335 & 0.510 & 0.897 \\
\hline Men, 19-64 years & $401 \cdot 0^{\mathrm{a}}$ & 5.95 & $410 \cdot 3^{\mathrm{a}}$ & 35.96 & $380 \cdot 5^{a}$ & $19 \cdot 81$ & $378.0^{\mathrm{a}}$ & $28 \cdot 11$ & 0.539 & 0.772 & 0.619 \\
\hline \multicolumn{12}{|l|}{ Iron (mg/1000 kcal) } \\
\hline Younger children, $1-8$ years & $6 \cdot 6^{a}$ & 0.09 & $7 \cdot 0^{\mathrm{a}}$ & 0.40 & $6 \cdot 8^{\mathrm{a}}$ & 0.31 & $6 \cdot 5^{\mathrm{a}}$ & 0.27 & 0.664 & 0.749 & 0.911 \\
\hline Older children, $9-18$ years & $6 \cdot 7^{\mathrm{a}}$ & 0.07 & $6 \cdot 5^{\mathrm{a}}$ & 0.26 & $6 \cdot 5^{\mathrm{a}}$ & 0.16 & $6 \cdot 1^{\mathrm{a}}$ & 0.21 & 0.078 & 0.278 & 0.092 \\
\hline Women, 19-64 years & $6 \cdot 9^{a}$ & 0.07 & $7 \cdot 0^{\mathrm{a}}$ & 0.32 & $6 \cdot 3^{\mathrm{a}}$ & 0.20 & $6 \cdot 8^{\mathrm{a}}$ & 0.34 & 0.060 & 0.357 & 0.919 \\
\hline Men, 19-64 years & $6 \cdot 6^{\mathrm{a}}$ & 0.06 & $6 \cdot 2^{\mathrm{a}}$ & 0.24 & $6 \cdot 3^{\mathrm{a}}$ & 0.24 & $6 \cdot 1^{\mathrm{a}}$ & 0.34 & 0.080 & 0.121 & 0.184 \\
\hline \multicolumn{12}{|l|}{$\mathrm{Mg}(\mathrm{mg} / 1000 \mathrm{kcal})$} \\
\hline Younger children, $1-8$ years & $152 \cdot 4^{\mathrm{a}}$ & 1.28 & $153 \cdot 0^{\mathrm{a}}$ & $6 \cdot 51$ & $131 \cdot 1^{b}$ & 2.40 & $148 \cdot 2^{\mathrm{a}}$ & 3.87 & $<.001$ & $<.001$ & 0.261 \\
\hline Older children, $9-18$ years & $141.9^{a}$ & 1.25 & $142 \cdot 6^{\mathrm{a}}$ & 4.54 & $138 \cdot 5^{\mathrm{a}}$ & 4.99 & $128 \cdot 9^{\mathrm{a}}$ & 5.46 & 0.043 & 0.227 & 0.039 \\
\hline Women, $19-64$ years & $180 \cdot 7^{a}$ & $2 \cdot 13$ & $175 \cdot 8^{\mathrm{a}}$ & 7.57 & $168 \cdot 1^{a, b}$ & $5 \cdot 71$ & $157 \cdot 3^{\mathrm{b}}$ & $5 \cdot 85$ & 0.001 & 0.064 & 0.008 \\
\hline \multirow{2}{*}{\multicolumn{12}{|c|}{ Phosphorous (mg/1000 kcal) }} \\
\hline & & & & & & & & & & & \\
\hline Younger children, $1-8$ years & $761 \cdot 3^{a}$ & 6.47 & $827 \cdot 0^{b}$ & $42 \cdot 12$ & $683.2^{c}$ & $19 \cdot 26$ & $755 \cdot 3^{a, b}$ & 23.20 & 0.003 & 0.007 & 0.513 \\
\hline Older children, $9-18$ years & $685 \cdot 6^{a}$ & $5 \cdot 80$ & $624 \cdot 3^{b}$ & $17 \cdot 28$ & $660 \cdot 4^{a, b}$ & $15 \cdot 77$ & $602 \cdot 0^{\mathrm{b}}$ & 21.73 & $<0.001$ & 0.030 & 0.086 \\
\hline Women, $19-64$ years & $711.8^{\mathrm{a}}$ & 7.56 & $693 \cdot 7^{\mathrm{a}}$ & $19 \cdot 74$ & $664 \cdot 0^{\mathrm{a}}$ & $17 \cdot 02$ & $677 \cdot 6^{\mathrm{a}}$ & 17.53 & 0.073 & 0.494 & 0.386 \\
\hline Men, $19-64$ years & $681 \cdot 1^{\mathrm{a}}$ & $5 \cdot 79$ & $688 \cdot 6^{a, b}$ & 33.07 & $632 \cdot 0^{\mathrm{b}}$ & $16 \cdot 32$ & $655 \cdot 1^{\mathrm{a}, \mathrm{b}}$ & 35.45 & 0.036 & 0.078 & 0.175 \\
\hline \multicolumn{12}{|l|}{ Potassium (mg/1000 kcal) } \\
\hline Younger children, $1-8$ years & $1498 \cdot 7^{\mathrm{a}}$ & $14 \cdot 21$ & $1469 \cdot 7^{a, b}$ & 51.92 & $1300 \cdot 7^{b}$ & $36 \cdot 39$ & $1399 \cdot 8^{a, b}$ & 64.31 & $<0.0001$ & 0.001 & 0.099 \\
\hline Older children, $9-18$ years & $1296 \cdot 0^{\mathrm{a}}$ & $11 \cdot 13$ & $1322 \cdot 3^{a}$ & 67.85 & $1243 \cdot 6^{a}$ & $46 \cdot 28$ & $1234.0^{\mathrm{a}}$ & 53.84 & 0.291 & 0.605 & 0.337 \\
\hline Women, $19-64$ years & $1569 \cdot 6^{a}$ & 18.93 & $1536 \cdot 2^{\mathrm{a}}$ & 54.29 & $1558 \cdot 7^{\mathrm{a}}$ & 47.67 & $1423 \cdot 1^{a}$ & 51.43 & 0.094 & 0.283 & 0.211 \\
\hline Men, $19-64$ years & $1434 \cdot 8^{\mathrm{a}}$ & $14 \cdot 19$ & $1454 \cdot 9^{\mathrm{a}}$ & 84.28 & $1362 \cdot 7^{\mathrm{a}}$ & $47 \cdot 11$ & $1343 \cdot 8^{a}$ & 74.64 & 0.053 & 0.524 & 0.414 \\
\hline \multicolumn{12}{|l|}{ Zinc (mg/1000 kcal) } \\
\hline Younger children, $1-8$ years & $5 \cdot 1^{\mathrm{a}}$ & 0.06 & $4.9^{\mathrm{a}}$ & 0.14 & $4 \cdot 8^{\mathrm{a}}$ & 0.14 & $4 \cdot 9^{\mathrm{a}}$ & $0 \cdot 19$ & 0.104 & 0.612 & 0.685 \\
\hline Older children, $9-18$ years & $5 \cdot 3^{\mathrm{a}}$ & 0.08 & $5 \cdot 0^{a, b}$ & 0.26 & $4.9^{\mathrm{a}}$ & 0.12 & $4.4^{b}$ & 0.25 & 0.001 & 0.024 & 0.006 \\
\hline Women, $19-64$ years & $5 \cdot 6^{\mathrm{a}}$ & 0.07 & $6 \cdot 0^{\mathrm{a}}$ & 0.34 & $5 \cdot 5^{\mathrm{a}}$ & 0.26 & $5 \cdot 3^{a}$ & 0.30 & 0.297 & 0.428 & 0.150 \\
\hline Men, $19-64$ years & $5 \cdot 8^{\mathrm{a}}$ & 0.08 & $5 \cdot 5^{a, b}$ & 0.27 & $5 \cdot 5^{b}$ & 0.22 & $5 \cdot 2^{b}$ & 0.36 & 0.110 & 0.022 & 0.017 \\
\hline
\end{tabular}

SEM, standard error of the mean; RAE, retinol activity equivalents; DFE, dietary folate equivalents.

*Separate models were run for each age-sex stratum. Means were derived from ANOVA, adjusted for age and sex (children's models only).

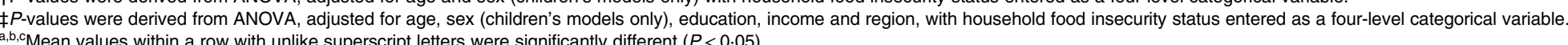


Zn per $1000 \mathrm{kcal}$ compared with the food secure (Table 5). Furthermore, men in severely food insecure households had significantly lower mean intakes of vitamin $\mathrm{C}$ and Zn per 1000 kcal compared with men in food-secure households (Table 5).

\section{Discussion}

Our findings suggest that household food insecurity status is a predictor of poorer diet quality among men, women, older children and younger children in Canada, even after taking household income, geographic region and education levels into account. Across all four groups, the mean proportion of total energy from ultra-processed foods was higher with more severe household food insecurity. This did not translate into higher carbohydrate, sugar, fat or saturated fat intakes or significant trends in HEI-C scores or fruit and vegetable intakes. However, $\mathrm{Na}$ intake/1000 kcal among younger children was positively associated with severity of household food insecurity. Although the specific micronutrients linked to food insecurity status differed across age/sex groups, the pattern was towards less nutrient dense dietary intakes among adults and children with more severe household food insecurity, with the most consistent results for men and older children.

Our observation of a graded relationship between the severity of household food insecurity and multiple indicators of diet quality among adults and older children is consistent with examinations of food insecurity status and dietary intakes in the $\mathrm{USA}^{(21,42)}$. The only similar trend observed among younger children was for Na/1000 kcal, but we found multiple indications of poorer diet quality among younger children in moderately food-insecure households. While it is possible that parents successfully buffered the effect of severe food insecurity on younger children's intakes ${ }^{(67)}$, our findings may also have been influenced by the small number of younger children in severely food-insecure households and by biases in the parental reporting of younger children's dietary intakes more generally ${ }^{(70)}$.

The poorer dietary quality among children 9-18 years with increasing severity of household food insecurity and the significantly poorer quality of dietary intakes among children 1-8 years in moderately food-insecure households suggest that the dietary intakes of children in Canada are affected by household food insecurity. This finding differs from earlier Canadian research showing moderate/severe household food insecurity to be more strongly associated with lower nutrient intakes and higher prevalence of nutrient inadequacies among adults than children ${ }^{(28)}$, but there are important methodologic differences between these two studies. Whereas we compared 1-d mean micronutrient intakes per $1000 \mathrm{kcal}$ to explore differences in diet quality, this earlier study compared absolute and usual nutrient intakes and the prevalence of inadequate nutrient intakes ${ }^{(28)}$. More analyses are needed to determine whether the nutritional vulnerability associated with household food insecurity in Canada has changed over time for different age groups.

We did not include nutrients from dietary supplements in our calculations of micronutrients per $1000 \mathrm{kcal}$, but the use of supplements is much lower among food-insecure than food-secure Canadians ${ }^{(38,71)}$. Given the evidence that supplement use contributes to nutrient adequacy ${ }^{(38)}$, particularly among more affluent individuals ${ }^{(72)}$, including nutrient intakes from supplements would likely have increased the observed differences between food-secure and food-insecure groups.

The heightened vulnerability associated with more severe food insecurity in the current study highlights the importance of not treating food insecurity as a simple binary variable when sample sizes are sufficient to permit more extensive analysis. We found no significant differences in the diet quality of adults in marginally food-insecure households compared with food-secure households and very few indications of differences among older children. Among younger children, a reverse association was observed for two micronutrients with marginal food insecurity associated with more nutrient dense intakes than food security. Marginal food security has been associated with significantly poorer diet quality and usual intakes for some nutrients among low-income adults in the $\mathrm{USA}^{(21,22)}$, but given Canada-US differences in the coding of the Household Food Security Survey Module, the marginal category in the US traverses the 'moderately food insecure' category applied in the current study. Marginal food insecurity, as it is defined in Canada, may not relate to dietary compromise in this country, but more comprehensive dietary assessment is required to reach this conclusion. Our analyses are based on a single day of intake data, with food insecurity measured retrospectively over the prior 12 months. An assessment of dietary intakes that extended over this same period might reveal more differences by food insecurity status.

Canada does not have large-scale publicly funded food assistance programmes like the USA, but smaller-scale, community programmes intended to improve fruit and vegetable access for vulnerable populations receive some public support ${ }^{(43,73)}$. This focus reflects the widely held belief that fruit and vegetable intakes are compromised in the context of household food insecurity, yet we found few differences in fruit and vegetable intakes by household food insecurity status. Household food insecurity status most clearly differentiated individuals in terms of their consumption of ultra-processed foods, a variable that has rarely been examined in relation to food insecurity. A positive association between food insecurity and the consumption of highly processed foods has also been reported in the $\mathrm{USA}^{(21)}$, but studies in Brazil have yielded mixed results ${ }^{(74,75)}$, perhaps reflecting differences in context, study design and measurements. The strong positive 
association between severity of household food insecurity and the proportion of energy derived from ultra-processed foods observed in our study among both adults and children is consistent with research pointing to the lower cost, higher satiety and greater availability of these foods ${ }^{(58,76)}$. A diet higher in unprocessed or minimally processed foods could be expected to cost more, putting it out of reach for people struggling to afford food and potentially heightening their risk of obesity ${ }^{(61,77)}$ and diet-related chronic diseases ${ }^{(59,78,79)}$. With the exception of $\mathrm{Na}$ intakes among younger children, we did not observe parallel trends for $\mathrm{Na}$, saturated fat or total sugar per $1000 \mathrm{kcal}$. It is important to note, however, that the available data precluded an examination of added sugars ${ }^{(50)}$. Higher intakes of added sugars have been associated with food insecurity among children ${ }^{(27)}$ and non-Hispanic white adults $^{(22)}$ in the USA; this should be investigated in Canada. As public health interventions to improve foodinsecure households' access to fresh fruits and vegetables continue to be launched ${ }^{(43)}$, research is also needed to determine how these programmes affect ultra-processed food consumption.

Strengths of the current study include the large sample size, examination of a wide spectrum of indicators of diet quality and use of a validated, standardised scale of severity to assess household food insecurity status. Our study also has several limitations. First, our comparisons of group means are based on a single 24-h recall. Although this provides a reasonably unbiased estimate of group mean usual intakes ${ }^{(69)}$, the high within-group variation in intakes based on one 24-h measure undoubtedly attenuated our ability to detect significant differences between groups. The collection of second 24-h recalls on only a third of the sample in CCHS 2015 Nutrition precluded the reliable estimation and analysis of usual intake distributions for adults and children by household food insecurity status. The paucity of second recalls in CCHS 2015 Nutrition limits the utility of this survey for analyses of vulnerable population subgroups compared with population surveys such as the US National Health and Nutrition Surveys that collect two 24-h recalls on all participants. Second, while we have charted several significant differences in group mean intakes by food insecurity status, more research is needed to determine the health implications of our findings. Specifically, population-representative studies with sufficient repeat measures of dietary intake to support the estimation of usual intakes are required to establish whether the differences in micronutrient density reported here translate into differences in risk of inadequate nutrient intakes. Studies also need to be conducted in Canada to examine the relationship between household food insecurity status and clinical and biochemical measures of nutritional status and cardiometabolic risk. Third, sample size limitations precluded analyses by race or Indigenous identity. Given the markedly higher odds of food insecurity among Black and Indigenous peoples in Canada ${ }^{(46,80)}$, race-based examinations of the nutritional implications of food insecurity are warranted. Finally, CCHS 2015-Nutrition omitted people living in the territories and in First Nations communities. While these represent relatively small fractions of the total population, they are at particularly high risk of food insecurity ${ }^{(1,46,81)}$. Research is needed to understand how household food insecurity relates to diet quality and other measures of nutritional compromise in these particularly vulnerable groups.

Our observation of graded relationships between the severity of household food insecurity and multiple indicators of diet quality among adults and children is consistent with US results ${ }^{(21,27,42)}$, and with Canadian research charting the much greater probability of morbidity and mortality among adults with more severe household food insecurity $^{(12,13,15,18)}$. The greater ultra-processed food consumption and lower nutrient density of dietary intakes among adults and children in more severely food insecure households also closely align with the significantly lower incomes ${ }^{(46)}$, lower food expenditures and more compromised household expenditure patterns in general ${ }^{(45)}$ and much more extreme financial hardship ${ }^{(47,82)}$ documented with increasing severity of household food insecurity in Canada. The poorer quality dietary intakes of adults and children in severely food-insecure households can be understood as one manifestation of the more pervasive material deprivation that characterises these households. More research is required to determine the full duration of individuals' exposures to moderate and severe household food insecurity and the short- and longer-term health implications of the associated dietary compromises, but it is important to recognise the potential for serious, negative consequences. A recent prospective cohort study in Ontario, Canada, found that adults' exposure to moderate/severe household food insecurity was associated with more than double the risk of subsequently developing type 2 diabetes ${ }^{(6)}$. Canada has no population-based survey that includes biochemical measures of nutritional status or disease biomarkers and an assessment of household food insecurity, but our results suggest a need for such research. Analyses of US data have revealed highly significant associations between food insecurity and nutrient deficiencies among children ${ }^{(83,84)}$, adolescents ${ }^{(85)}$ and adults ${ }^{(86)}$, as well as associations with biomarkers of chronic disease ${ }^{(87-90)}$ and cumulative biological risk ${ }^{(91)}$.

In conclusion, our findings suggest that household food insecurity status is an important marker of poor dietary intakes in Canada, raising questions about the nutritional status and present and future health of people living in food-insecure households. Our results add to the much broader body of literature in Canada documenting the poorer health and well-being of adults and children in food-insecure households. When considered in this larger context, our findings do not imply a need for nutrition intervention so much as policy interventions to address the underlying causes of household food insecurity. Just as research internationally has highlighted the importance 
of investments in social protection programmes ${ }^{(92,93)}$ and more generous wage setting policies ${ }^{(94)}$ in reducing food insecurity rates, studies in Canada have charted the tight intersection between food insecurity and income support policies and labour market conditions in this country. Specifically, research on social assistance ${ }^{(95,96)}$, child benefits $^{(97-99)}$, minimum wages ${ }^{(96)}$, income tax thresholds ${ }^{(96)}$ and public pensions ${ }^{(51)}$ suggest that policies that support more adequate, secure incomes for low-income households are key to reducing the prevalence and severity of household food insecurity in Canada.

\section{Acknowledgements}

Acknowledgements: The authors are indebted to Claire LaMantia for assistance with the data analysis. Financial support: The current study was supported by the Canadian Institutes of Health Research (FRN 151526). Conflicts of interest: The authors have no conflicts of interests. Authorship: V.T. and J.H. conceptualised and designed the current study. J.H. conducted the data analyses. Both authors participated in the data interpretation and writing of this manuscript. Etbics of human subject participation: This was a secondary analysis of population survey data, so the Ethical Standards Disclosure is not applicable. The study was approved by the Health Sciences Research Ethics Board at University of Toronto.

\section{References}

1. Tarasuk V \& Mitchell A (2020) Household Food Insecurity in Canada, 2017-2018. Toronto, ON: Research to Identify Policy Options to Reduce Food Insecurity.

2. Statistics Canada (2020) Food Insecurity During the COVID-19 Pandemic, May 2020. Statistics Canada. https:// www150.statcan.gc.ca/n1/pub/45-28-0001/2020001/article/ 00039-eng.htm (accessed June 2020).

3. Kirkpatrick S, McIntyre L \& Potestio M (2010) Child hunger and long-term adverse consequences for health. Arch Pediatr Adolesc Med 164, 754-762.

4. McIntyre L, Wu X, Kwok C et al. (2017) The pervasive effect of youth self-report of hunger on depression over 6 years of follow up. Soc Psychiatry Psychiatr Epidemiol 52, 537-547.

5. Cox J, Hamelin AM, McLinden T et al. (2016) Food insecurity in HIV-hepatitis C virus co-infected individuals in Canada: the importance of co-morbidities. AIDS Behav 21, 792-802.

6. Tait C, L'Abbe M, Smith P et al. (2018) The association between food insecurity and incident type 2 diabetes in Canada: a population-based cohort study. PLoS One 13, $\mathrm{e} 0195962$.

7. Vozoris N \& Tarasuk V (2003) Household food insufficiency is associated with poorer health. J Nutr 133, 120-126.

8. Maynard M, Andrade L, Packull-McCormick S et al. (2018) Food insecurity and mental health among females in highincome countries. Int J Environ Res Public Health 15, 1-36.

9. Muldoon KA, Duff PK, Fielden S et al. (2012) Food insufficiency is associated with psychiatric morbidity in a nationally representative study of mental illness among food insecure Canadians. Soc Psychiatry Psychiatr Epidemiol 48, 795-803.
10. Anema A, Chan K, Weiser S et al. (2013) Relationship between food insecurity and mortality among HIV-positive injection drug users receiving antiretroviral therapy in British Columbia, Canada. PLoS One 8, e61277.

11. Gucciardi E, DeMelo M, Vogt J et al. (2009) Exploration of the relationship between household food insecurity and diabetes care in Canada. Diabetes Care 32, 2218-2224.

12. Jessiman-Perreault G \& McIntyre L (2017) The household food insecurity gradient and potential reductions in adverse population mental health outcomes in Canadian adults. SSM Popul Health 3, 464-472.

13. Tarasuk V, Mitchell A, McLaren L et al. (2013) Chronic physical and mental health conditions among adults may increase vulnerability to household food insecurity. J Nutr 143, 1785-1793.

14. Men F, Elgar F \& Tarasuk V (2021) Food insecurity is associated with mental health problems among Canadian youth. $J$ Epidemiol Community Health 75, 741-748.

15. Men F, Gundersen C, Urquia ML et al. (2020) Food insecurity is associated with higher health care use and costs among Canadian adults. Health Aff 39, 1377-1385.

16. Tarasuk V, Cheng J, Gundersen C et al. (2018) The relation between food insecurity and mental health service utilization in Ontario. Can J Psychiatry 63, 557-569.

17. Tarasuk V, Cheng J, de Oliveira C et al. (2015) Association between household food insecurity and annual health care costs. Can Med Assoc J 187, E429-E436.

18. Men F, Gundersen C, Urquia ML et al. (2020) Association between household food insecurity and mortality in Canada: a population-based retrospective cohort study. Can Med Assoc J 192, E53-E60.

19. Gundersen C, Tarasuk V, Cheng L et al. (2018) Food insecurity status and mortality among adults in Ontario, Canada. PLoS One 13, e0202642.

20. Hanson K \& Connor L (2014) Food insecurity and dietary quality in US adults and children: a systematic review. $\mathrm{Am}$ J Clin Nutr 100, 684-692.

21. Leung C, Epel E, Ritchie L et al. (2014) Food insecurity is inversely associated with diet quality of lower-income adults. J Acad Nutr Diet 114, 1943-1953.

22. Leung C \& Tester J (2019) The association between food insecurity and diet quality varies by race/ethnicity: an analysis of National Health and Nutrition Examination Survey 2011-2014 Results. J Acad Nutr Diet 119, 1676-1686.

23. Luciana de Araujo M, Mendonca R, Filho J et al. (2018) Association between food insecurity and food intake. Nutrition 54, 54-59.

24. Kirk S, Kuhle S, McIsaac J et al. (2014) Food security status among grade 5 students in Nova Scotia, Canada and its associations with health outcomes. Public Health Nutr 18, $2943-2951$.

25. Landry M, van den Berg A, Asigbee F et al. (2019) Child-report of food insecurity is associated with diet quality in children. Nutrients 11, 1574.

26. Rossen L \& Kobernik E (2015) Food insecurity and dietary intake among US youth, 2007-2010. Pediatr Obes 11, 187-193.

27. Eicher-Miller H \& Zhao Y (2018) Evidence of the age-specific relationship of food insecurity and key dietary outcomes among US children and adolescents. Nutr Res Rev 31, 98-113.

28. Kirkpatrick S \& Tarasuk V (2008) Food insecurity is associated with nutrient inadequacies among Canadian adults and adolescents. J Nutr 138, 604-612.

29. Kirkpatrick SI, Dodd KW, Parsons R et al. (2015) Household food insecurity is a stronger marker of adequacy of nutrient intakes among Canadian compared to American youth and adults. J Nutr 145, 1596-1603.

30. Vatanparast H, Calvo M, Green TJ et al. (2010) Despite mandatory fortification of staple foods, vitamin D intakes 
of Canadian children and adults are inadequate. J Steroid Biochem Mol Biol 121, 301-303.

31. Mark S, Lambert M, O'Loughlin J et al. (2012) Household income, food insecurity and nutrition in Canadian youth. Can J Public Health 103, 94-99.

32. Nshimyumukiza L, Lieffers J, Ekwaru J et al. (2018) Temporal changes in diet quality and the associated economic burden in Canada. PLoS One 13, e0206877.

33. Czoli C, Jones A \& Hammond D (2019) Trends in sugary drinks in Canada, 2004 to 2015: a comparison of market sales and dietary intake data. Public Health Nutr 22, 2723-2728.

34. Tugault-Lafleur C \& Black J (2019) Differences in the quantity and types of foods and beverages consumed by Canadians between 2004 and 2015. Nutrients 11, 526.

35. Tugault-Lafleur C, Barr S \& Black J (2019) Examining differences in school hour and school day dietary quality among Canadian children between 2004 and 2015. Public Health Nutr 22, 3051-3062.

36. Polsky JY \& Garriguet D (2020) Change in vegetable and fruit consumption in Canada between 2004 and 2015. Health Rep 31, 3-12.

37. Tugault-Lafleur CN \& Black JL (2020) Lunch on school days in Canada: examining contributions to nutrient and food group intake and differences across eating locations. J Acad Nutr Diet 120, 1484-1497.

38. Vatanparast H, Islam N, Patil R et al. (2020) Calcium intake from food and supplemental sources decreased in the Canadian population from 2004 to 2015. J Nutr 150, 833-841.

39. Hosseini S, Jones J \& Vatanparast H (2019) Association between grain intake, nutrient intake, and diet quality of Canadians: evidence from the Canadian community health survey - nutrition. Nutrients 11, 1937.

40. Hosseini S, Papanikolaou Y, Islam N et al. (2019) Consumption patterns of grain-based foods among adults in Canada: evidence from the Canadian community health survey - nutrition 2015. Nutrients 11, 784.

41. Vatanparast H, Islam N, Shafiee M et al. (2020) Increasing plant-based meat alternatives and decreasing red and processed meat in the diet differentially affect the diet quality and nutrient intakes of Canadians. Nutrients 12, 2034.

42. Jun S, Zeh M, Eicher-Miller H et al. (2019) Children's dietary quality and micronutrient adequacy by food security in the household and among household children. Nutrients 11,965.

43. Government of Canada (2020) Government of Canada Supports Initiative to Help Canadians with Low Incomes Access Fresh Produce. Ottawa, ON: Government of Canada.

44. Men F \& Tarasuk V (2020) Severe food insecurity associated with mortality among lower-income Canadian adults approaching eligibility for public pensions: a population cohort study. BMC Public Health 20, 1484.

45. Fafard St-Germain AA \& Tarasuk V (2018) Prioritization of the essentials in the spending patterns of Canadian households experiencing food insecurity. Public Health Nutr 21, 2065-2078.

46. Tarasuk V, Fafard St-Germain AA \& Mitchell A (2019) Geographic and socio-demographic predictors of household food insecurity in Canada, 2011-2012. BMC Public Health 19, $1-12$.

47. Men F, Gundersen C, Urquia ML et al. (2019) Prescription medication non-adherence associated with food insecurity: a population-based cross-sectional study. CMAJ Open 7, E590-E597.

48. Loopstra R \& Tarasuk V (2013) What does increasing severity of food insecurity indicate for food insecure families? Relationship between severity of food insecurity and indicators of material hardship and constrained food purchasing. J Hunger Environ Nutr 8, 337-349.
49. Government of Canada (2020) Determining Food Security Status. https://www.canada.ca/en/health-canada/services/ food-nutrition/food-nutrition-surveillance/health-nutritionsurveys/canadian-community-health-survey-cchs/householdfood-insecurity-canada-overview/determining-food-secu rity-status-food-nutrition-surveillance-health-canada.html (accessed July 2020).

50. Health Canada (2017) Reference Guide to Understanding and Using the Data. 2015 Canadian Community Health Survey - Nutrition. Ottawa, ON: Health Canada.

51. McIntyre L, Dutton D, Kwok C et al. (2016) Reduction of food insecurity in low-income Canadian seniors as a likely impact of a guaranteed annual income. Can Public Policy 42, 274-286.

52. Health Canada (2007) Canadian Community Health Survey, Cycle 2.2, Nutrition (2004) - Income-Related Household Food Security in Canada. Ottawa: Office of Nutrition Policy and Promotion, Health Products and Food Branch, Health Canada.

53. Hamilton W, Cook J, Thompson W et al. (1997) Household Food Security in the United States in 1995. Technical Report of the Food Security Measurement Project. Alexandria, VA: United States Department of Agriculture.

54. Health Canada (2019) Canada's Dietary Guidelines. Ottawa, ON: Health Canada.

55. Garriguet D (2009) Diet quality in Canada. Health Rep 20, 1-12.

56. Health Canada (2007) Eating Well with Canada's Food Guide (2007). Ottawa, ON: Health Canada.

57. Moubarac J, Batal M, Louzada M et al. (2017) Consumption of ultra-processed foods predict diet quality in Canada. Appetite 108, 512-520.

58. Monteiro C, Cannon G, Moubarac J et al. (2017) The UN decade of nutrition, the NOVA food classification and the trouble with ultra-processing. Public Health Nutr 21, 5-17.

59. Paglia G, Dinu M, Madarena M et al. (2021) Consumption of ultra-processed foods and health status: a systematic review and meta-analysis. Br J Nutr 125, 308-318.

60. Elizabeth L, Machado P, Zinocker M et al. (2020) Ultra-processed foods and health outcomes: a narrative review. Nutrients 12, 1955

61. Nardocci M, Polsky JY \& Moubarac J (2021) Consumption of ultra-processed foods is associated with obesity, diabetes and hypertension in Canadian adults. Can J Public Health 112, 421-429.

62. Danyliw A, Vatanparast H, Nikpartow N et al. (2011) Beverage intake patterns of Canadian children and adolescents. Public Health Nutr 14, 1961-1969.

63. Nikpartow N, Danyliw A, Whiting S et al. (2012) Beverage consumption patterns of Canadian adults aged 19 to 65 years. Public Health Nutr 15, 2175-2184.

64. Tarasuk V, Fitzpatrick S \& Ward H (2010) Nutrition inequities in Canada. Appl Physiol Nutr Metab 38, 172-179.

65. Health Canada \& Statistics Canada (2008) Canadian Community Health Survey, Cycle 2.2, Nutrition, 2004 Nutrient Intakes from Food. Provincial, Regional, and National Summary Data Tables, Volume 3. Ottawa, ON: Health Canada, Statistics Canada.

66. Health Canada \& Statistics Canada (2008) Canadian Community Health Survey, Cycle 2.2, Nutrition, 2004 Nutrient Intakes from Food. Provincial, Regional, and National Summary Data Tables, Volume 2. Ottawa, ON: Health Canada, Statistics Canada.

67. McIntyre L, Glanville T, Raine KD et al. (2003) Do lowincome lone mothers compromise their nutrition to feed their children? Can Med Assoc J 168, 686-691.

68. Glanville NT \& McIntyre L (2006) Diet quality of Atlantic families headed by single mothers. Can J Diet Prac Res $\mathbf{6 7}$, $28-35$. 
69. Institute of Medicine (2000) Dietary Reference Intakes: Applications in Dietary Assessment. Washington, DC: National Academy Press.

70. Montgomery C, Reilly J, Jackson D et al. (2005) Validation of energy intake by 24-h multiple pass recall: comparison with total energy expenditure in children aged 5-7 years. BrJ Nutr 93, 671-676.

71. Vatanparast H, Patil R, Islam N et al. (2020) Vitamin D intake from supplemental sources but not food sources has increased in the Canadian population over time. $J$ Nutr 150, 526-535.

72. Blumberg J, Frei B, Fulgoni V et al. (2018) Contribution of dietary supplements to nutritional adequacy by socioeconomic subgroups in adults in the United States. Nutrients 10, 1-11.

73. Population and Public Health \& BC Ministry of Health (2014) Model Core Program Paper: Food Security. http://www2. gov.bc.ca/assets/gov/health/about-bc-s-health-care-system/ public-health/healthy-living-and-healthy-communities/food_ security_model_core_program_paper.pdf (accessed May 2017).

74. de Araujo M, Mendonca R, Filho J et al. (2018) Association between food insecurity and food intake. Nutrients $\mathbf{5 4}$, 54-59.

75. Sato P, Ulian M, Oliveira M et al. (2020) Signs and strategies to deal with food insecurity and consumption of ultraprocessed foods among Amazonian mothers. Glob Public Health 15, 1130-1143.

76. Moubarac J, Bortoletto Martins A, Claro R et al. (2012) Consumption of ultra-processed foods and likely impact on human health. Evidence from Canada. Public Health Nutr 16, 2240-2248.

77. Drewnowski A, Darmon N \& Briend A (2004) Replacing fats and sweets with vegetables and fruit - a question of cost. $\mathrm{Am}$ J Public Health 94, 1555-1559.

78. Srour B, Fezeu L, Kesse-Guyot E et al. (2019) Ultra-processed food intake and risk of cardiovascular disease: prospective cohort study. BMJ 365, I1451.

79. Costa de Miranda R, Rauber F \& Levy R (2021) Impact of ultra-processed food consumption on metabolic health. Curr Opin Lipidol 31, 24-37.

80. Dhunna S \& Tarasuk V (2021) Black-white racial disparities in household food insecurity from 2005-2014, Canada. Can J Public Health, 1-15. doi: 10.17269/s41997-021-00539-y.

81. Deaton B, Scholz A \& Lipka B (2020) An empirical assessment of food security on First Nations in Canada. Can J Agric Econ 68, 5-19.

82. Loopstra R \& Tarasuk V (2013) What does increasing severity of food insecurity indicate for food insecure families? Relationships between severity of food insecurity and indicators of material hardship and constrained food purchasing. J Hunger Environ Nutr 8, 337-349.

83. Skalicky A, Meyers A, Adams W et al. (2006) Child food insecurity and iron deficiency anemia in low-income infants and toddlers in the United States. Matern Child Health J 10, $177-185$.

84. Eicher-Miller H, Mason A, Weaver C et al. (2011) Food insecurity is associated with diet and bone mass disparities in early adolescent males but not females in the United States. J Nutr 141, 1738-1745.

85. Eicher-Miller H, Mason A, Weaver C et al. (2009) Food insecurity is associated with iron deficiency anemia in US adolescents. Am J Clin Nutr 90, 1358-1371.

86. Park C \& Eicher-Miller H (2014) Iron deficiency is associated with food insecurity in pregnant females in the United States: National Health and Nutrition Examination Survey 1999-2010. J Acad Nutr Diet 114, 1967-1973.

87. Seligman HK, Jacobs E, Lopez A et al. (2011) Food insecurity and hypoglycemia among safety net patients with diabetes. Arch Intern Med 171, 1204-1206.

88. Ford E (2013) Food security and cardiovascular disease risk among adults in the United States: findings from the National Health and Nutrition Examination Survey, 2003-2008. Prev Chronic Dis 10, 130244.

89. Parker E, Widome R, Nettleton J et al. (2010) Food security and metabolic syndrome in U.S. adults and adolescents: findings from the National Health and Nutrition Examination Survey, 1996-2006. Ann Epidemiol 20, 364-370.

90. Tester J, Laraia BA, Leung C et al. (2016) Dyslipidemia and food security in low-income US adolescents: National Health and Nutrition Examination Survey, 2003-2010. Prev Chronic Dis 13, 150441.

91. Leung CW \& Zhou M (2020) Household food insecurity and the association with cumulative biological risk among lower-income adults: results from the National Health and Nutrition Examination Surveys 2007-2010. Nutrients 12, 1517.

92. Loopstra R, Reeves A, McKee M et al. (2016) Food insecurity and social protection in Europe: quasi-natural experiment of Europe's great recessions 2004-2012. Prev Med 89, 44-50.

93. Fernald L \& Gosliner W (2019) Alternatives to SNAP: global approaches to addressing childhood poverty and food insecurity. Am J Public Health 109, 1668-1677.

94. Reeves A, Loopstra R \& Tarasuk V (2021) Wage setting policies, employment, and food insecurity: a multilevel analysis of 492,078 people in 139 countries. Am J Public Health 111, $718-725$.

95. Loopstra R, Dachner N \& Tarasuk V (2015) An exploration of the unprecedented decline in the prevalence of household food insecurity in Newfoundland and Labrador, 2007-2012. Can Public Policy 41, 191-206.

96. Men F, Urquia ML \& Tarasuk V (2021) The role of provincial social policies and economic environment in shaping household food insecurity among families with children in Canada. Prev Med 148, 106558.

97. Tarasuk V, Li N, Dachner N et al. (2019) Household food insecurity in Ontario during a period of poverty reduction, 2005-2014. Can Public Policy 45, 93-104.

98. Ionescu-Ittu R, Glymour M \& Kaufman J (2015) A difference-in-difference approach to estimate the effect of income-supplementation on food insecurity. Prev Med 70, 108-116.

99. Brown E \& Tarasuk V (2019) Money speaks: reductions in severe food insecurity follow the Canada child benefit. Prev Med 129, 105876. 